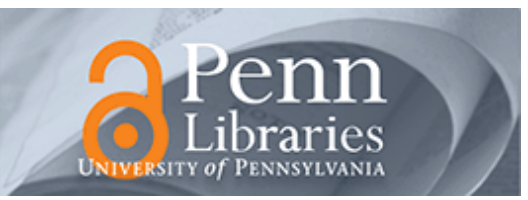

University of Pennsylvania

ScholarlyCommons

4-1-2009

\title{
Integration of AIN Micromechanical Contour-Mode Technology Filters with Three-Finger Dual Beam AIN MEMS Switches
}

\author{
Nipun Sinha \\ University of Pennsylvania, nipun@seas.upenn.edu \\ Rashed Mahameed \\ University of California, San Diego \\ Chengjie Zuo \\ University of Pennsylvania, chengjiezuo@hotmail.com \\ Gianluca Piazza \\ University of Pennsylvania, piazza@seas.upenn.edu
}

Follow this and additional works at: https://repository.upenn.edu/ese_papers

Part of the Electro-Mechanical Systems Commons

\section{Recommended Citation}

Nipun Sinha, Rashed Mahameed, Chengjie Zuo, and Gianluca Piazza, "Integration of AIN Micromechanical Contour-Mode Technology Filters with Three-Finger Dual Beam AIN MEMS Switches", 2009 Joint Meeting of the European Frequency and Time Forum and the IEEE International Frequency Control Symposium (EFTF-IFCS 2009) , 1-4. April 2009.

Suggested Citation:

Nipun Sinha, Rashed Mahameed, Chengjie Zuo, and Gianluca Piazza. "Integration of AIN Micromechanical ContourMode Technology Filters with Three-Finger Dual Beam AIN MEMS Switches" 2009 Joint Meeting of the European Frequency and Time Forum and the IEEE International Frequency Control Symposium (EFTF-IFCS 2009) (2009): 1-4. (C)20xx IEEE. Personal use of this material is permitted. However, permission to reprint/republish this material for advertising or promotional purposes or for creating new collective works for resale or redistribution to servers or lists, or to reuse any copyrighted component of this work in other works must be obtained from the IEEE.

This paper is posted at ScholarlyCommons. https://repository.upenn.edu/ese_papers/541

For more information, please contact repository@pobox.upenn.edu. 


\title{
Integration of AIN Micromechanical Contour-Mode Technology Filters with Three- Finger Dual Beam AIN MEMS Switches
}

\author{
Abstract \\ In this paper, we present the first demonstration of the monolithic integration of Aluminum Nitride (AIN) \\ micromechanical contour mode technology filters with dual-beam actuated MEMS AIN switches. This \\ integration has lead to the development of the first prototype of a fully-integrated all-mechanical \\ switchable filter. Integration has been demonstrated by using AIN contour-mode MEMS filters at two \\ center frequencies, i.e. 98.7 and $279.9 \mathrm{MHz}$. The micromechanical switch design used here is a novel \\ three-finger dual-beam topology that improves the isolation and insertion loss of the switch by decreasing \\ the parasitic coupling between the DC and RF signals over a previous AIN MEMS dual-beam design. With \\ the use of just one switch fabricated right next to, and integrated with the filter, the AIN MEMS filter is \\ effectively turned off and its pass-band transmission is lowered to the out of band level at $279.9 \mathrm{MHz}$. \\ Disciplines \\ Electro-Mechanical Systems

\section{Comments} \\ Suggested Citation: \\ Nipun Sinha, Rashed Mahameed, Chengjie Zuo, and Gianluca Piazza. "Integration of AIN Micromechanical \\ Contour-Mode Technology Filters with Three-Finger Dual Beam AIN MEMS Switches" 2009 Joint Meeting \\ of the European Frequency and Time Forum and the IEEE International Frequency Control Symposium \\ (EFTF-IFCS 2009) (2009): 1-4. \\ (C20xx IEEE. Personal use of this material is permitted. However, permission to reprint/republish this \\ material for advertising or promotional purposes or for creating new collective works for resale or \\ redistribution to servers or lists, or to reuse any copyrighted component of this work in other works must \\ be obtained from the IEEE.
}




\section{Integration of AlN Micromechanical Contour-Mode Technology Filters with Three-Finger Dual Beam AIN MEMS Switches}

\author{
Nipun Sinha \\ Mechanical Engineering and Applied Mechanics \\ University of Pennsylvania \\ Philadelphia, USA \\ nipun@seas.upenn.edu
}

\author{
Rashed Mahameed, Chengjie Zuo and Gianluca Piazza \\ Electrical and Systems Engineering \\ University of Pennsylvania \\ Philadelphia, USA \\ \{mrashed, czuo,piazza $@$ @seas.upenn.edu
}

\begin{abstract}
In this paper, we present the first demonstration of the monolithic integration of Aluminum Nitride (AIN) micromechanical contour mode technology filters with dualbeam actuated MEMS AIN switches. This integration has lead to the development of the first prototype of a fully-integrated allmechanical switchable filter. Integration has been demonstrated by using AIN contour-mode MEMS filters at two center frequencies, i.e. 98.7 and $279.9 \mathrm{MHz}$. The micromechanical switch design used here is a novel three-finger dual-beam topology that improves the isolation and insertion loss of the switch by decreasing the parasitic coupling between the DC and RF signals over a previous AIN MEMS dual beam design. With the use of just one switch fabricated right next to, and integrated with the filter, the AIN MEMS filter is effectively turned off and its pass-band transmission is lowered to the out of band level at 279.9 MHz.
\end{abstract}

\section{INTRODUCTION}

Miniaturization along with the development of low-loss and low-power devices have been one of the main drivers for both academic and industry research in the space of Radio Frequency (RF) communications. As demonstrated by previous implementation, there is always a trade-off between the goal of having a low-loss and low-power device and occupying a small footprint. The primary reason for this trade-off has been the use of different technologies, which require bonding, stacking or packaging of different components and comes at a significant price in terms of space, performance and cost. This trade-off will, in the near future, become a bottleneck for the implementation of multifrequency and multi-band wireless architectures. The implementation of these new classes of transceivers will require the realization of a single-chip multi-frequency highlyreconfigurable system capable of low power and low loss operation in a miniaturized fashion. The easiest and most elegant way to achieve such integration is to develop a technology platform that can implement all of the required components on a single chip. Aluminum Nitride (AlN) based piezoelectric contour mode technology has the potential to achieve such system level integration (with filters, resonators and switches that can be directly manufactured on CMOS) in the near future. The demonstration presented in this paper is the first step towards that ultimate solution.

AlN contour-mode filters [1]-[4] and resonators [5]-[7] have already been successfully demonstrated in the past. Contour-mode technology provides numerous advantages like, the ability to attain a single-chip multi-frequency solution in a small form factor, achieve narrow bandwidth and provide high off-band rejection. Filters have been demonstrated with center frequency ranging from $94 \mathrm{MHz}$ [3] up to approximately $3 \mathrm{GHz}$ [4]. This technology has been able to show a wide frequency range of operation and the development of a multifrequency solution is definitely possible. Nonetheless, a multifrequency system requires the ability to select a specific frequency of operation; i.e. there is a need for a switchable filter bank. To achieve this goal, while maintaining low losses and small form factor, the best option is to integrate the filter with RF MEMS switches.

RF MEMS switches have received considerable attention over the last two decades as they provide advantages like higher isolation, lower insertion loss and lower power consumption over traditional CMOS pin-diodes and FETs [8]. RF MEMS switches are therefore the best candidates for the implementation of large switched matrices of RF components, for which low power consumption is a requirement. Integration of filters with RF MEMS switches can lead to the next level of miniaturization and will minimize interconnect losses as the industry moves towards a system-on-chip solution. Integration will also open the development of high frequency RF solutions, like fully-mechanical transceivers that will operate in the microwave range, and which would have otherwise been hindered by parasitics.
This work was sponsored by the Defense Advanced Research Projects Agency (DARPA) and the National Science Foundation (NSF). 
Many transduction mechanisms have been used for the development of RF MEMS switches. Electrostatic actuation has been the most commonly used amongst them. Electrostatic actuation suffers from the drawback of requiring high actuation voltages. On the other hand, the switches that have been developed using piezoelectric actuation, using either Lead Zirconate Titanate (PZT) [9]-[10] or AlN [11]-[12], have shown comparatively lower voltages. In this work, we limited the number of different materials in the process and used an AlN based three-finger dual-beam switch for the development of a switchable filter based on AlN contour-mode technology. This was done with the aim of keeping the actuation voltages low and in line with modern supplies scaling and the fabrication process as simple as possible and CMOS compatible.

S-parameter cascading of co-fabricated AIN switches and resonators [11]-[12] have already shown that mechanical elements can be used for switching a resonator on and off. Pulskamp et al. [10] have also demonstrated the integration of a PZT based single pole dual throw (SP2T) switch with PZT based contour-mode filters. PZT has the disadvantage of not being CMOS compatible and hard to work with. AlN based processes are instead CMOS compatible and AIN is already a proven material for RF applications (see Avago FBAR [13]). In this paper we present the monolithic integration of AIN based contour-mode filters with an AlN based three-finger dual-beam actuation switch (Fig. 1).

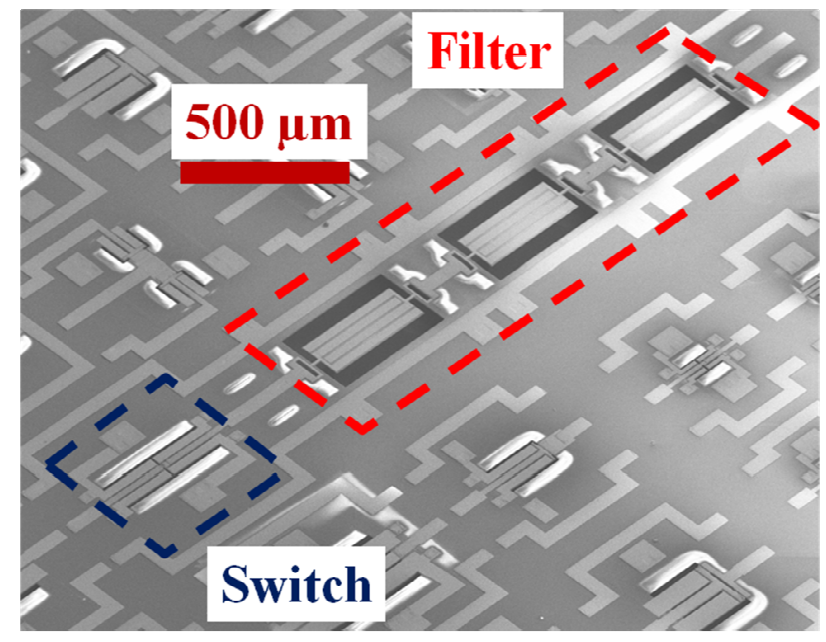

Figure 1. SEM of an AlN based three-finger dual-beam actuation switch integrated with an AlN contour-mode filter.

\section{DESIGN}

The design of the switch and the filter needs to take into account the limitations that each device poses on the other when integrated. The biggest hurdle of material compatibility was dealt with by using the same functional material, AlN, for both the switch and the filter. The second challenge was to force the AlN switch to use the same AlN thickness that is employed for the making of the filter. Finally, low loss in both devices was achieved by separately optimizing the layout of the two structures.

\section{A. Switch Design}

A new three-finger dual-beam actuation switch was used for achieving low loss integration with the filter. This design greatly reduces the parasitic coupling between the DC and RF lines that existed in the previous dual-beam design, by separating the actuation and RF lines (Fig. 2). The outer two fingers of the three-finger design are used for actuation whereas the central finger is used for carrying the RF signal.

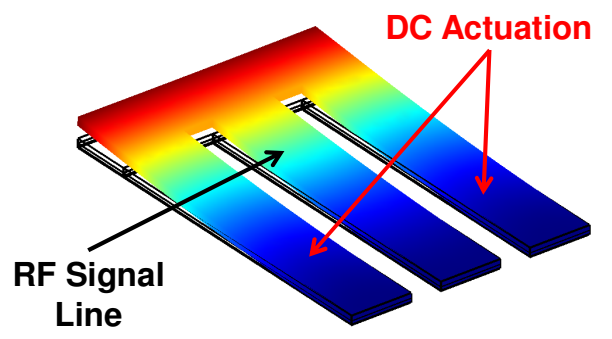

Figure 2. 3-D view of one of the beams that make up the three-finger dualbeam actuation switch. The figure shows the division of the beam into two DC actuation beams and the RF signal line.

The working principle (shown in Fig. 3) of the switch is very similar to the dual-beam actuation switch that had been developed earlier [11]-[12]. In this novel design bimorph actuation (two actuation layers) can be implemented, whereas solely unimorph actuation (single layer actuation) was possible in the previous design in which the actuator and the RF signal were stacked on top of each other. As shown in Fig. 3, the cross-section of the actuating beams consists of two layers of AlN that are sandwiched between three layers of Platinum. The routing of the electrodes is done in such a manner that both the opposing beams are simultaneously actuated in opposite directions by reversing the polarity of the applied electric field.
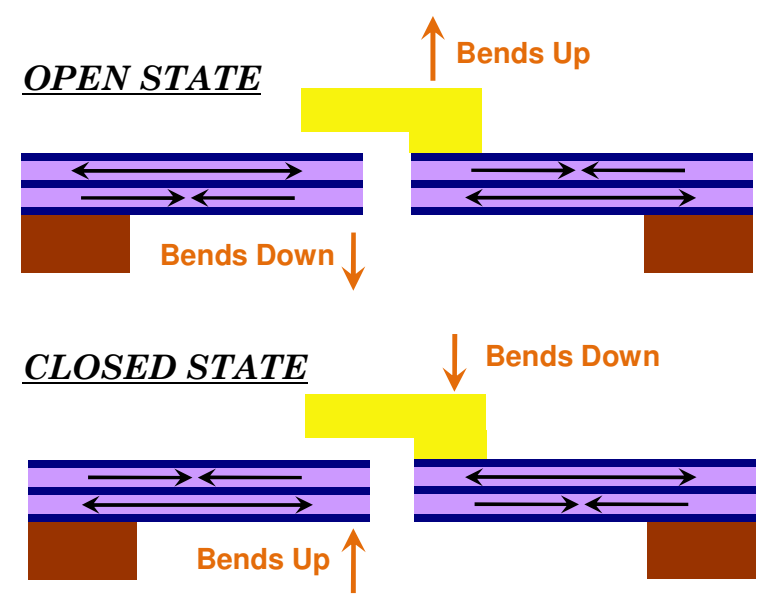

Figure 3. A 2-D cross-section of the actuation beam of the three-finger switch design. The schematic shows the basic operating principle of a bimorph actuator. The contact tip is present exclusively on the RF signal line and is drawn just for illustrating the operating principle of the switch (the gold line is not in the same plane of the bimorph actuator).

\section{B. Filter Design}

For verification of the switch-filter integration process and direct comparison with previous results, the adopted filter 
design is based on the one published by Zuo et al. [3]. The filter is a $3^{\text {rd }}$ order element composed of three two-port piezoelectric AlN contour-mode resonators. The two-port AlN resonators are directly cascaded and electrically coupled by the intrinsic capacitance existing in the piezoelectric transducers, so that no external coupling components are needed to realize higher-order filtering. This solution was chosen over a more traditional ladder topology because it is in line with the goals of miniaturization and ease of integration. In fact, compared with a ladder topology, the self-coupling scheme provides narrower bandwidth and better rejection, reduces overall device size by employing fewer components, and improves manufacturing yield by using single-frequency resonators.

\section{FABRICATION}

The integrated fabrication process for the switch and the filter builds upon the well established 4-mask fabrication process for contour-mode filters [1]. The switch process (Fig. 4) is an 8-mask post-CMOS compatible process that is very similar to the one described in [11]-[12]. The addition of a mask, with respect to the previous process, is for the planarization of the contact tip over the etch pit by using photoresist as the refill layer. This was done to improve the yield of the fabrication process.

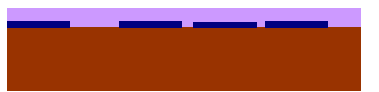

(a)

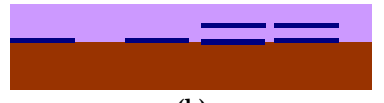

(b)

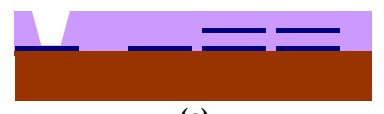

(c)

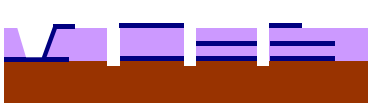

(d)

$\square$ Si $\square$ Pt AlN Si

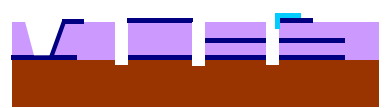

(e)

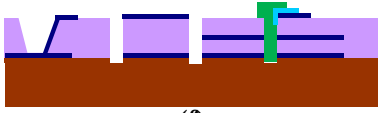

(f)

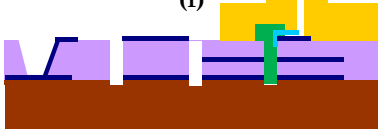

(g)

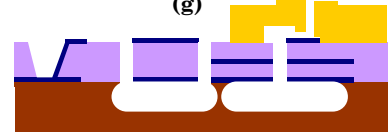

(h)
Refill Layer Gold

Figure 4. 8-mask post-CMOS compatible switch fabrication process.

\section{EXPERIMENTAL RESULTS}

In order to quantify the impact of the additional fabrication steps on the filter performance, direct measurement of individual filters fabricated with the switches were done and compared to previously made filters without the switch. 2-port measurements of the filters were performed using an Agilent N5230 PNA-L Network Analyzer. The measurements show that, despite the increase in the number of steps in the fabrication process, the filters were able to maintain the same level of performance that had been achieved without the switch. Fig. 5 shows the experimental response of a $98.7 \mathrm{MHz}$ third-order filter fabricated with this 8 mask process. This filter shows insertion loss and rejection that are similar to what has been achieved for a similar structure in the past [3].

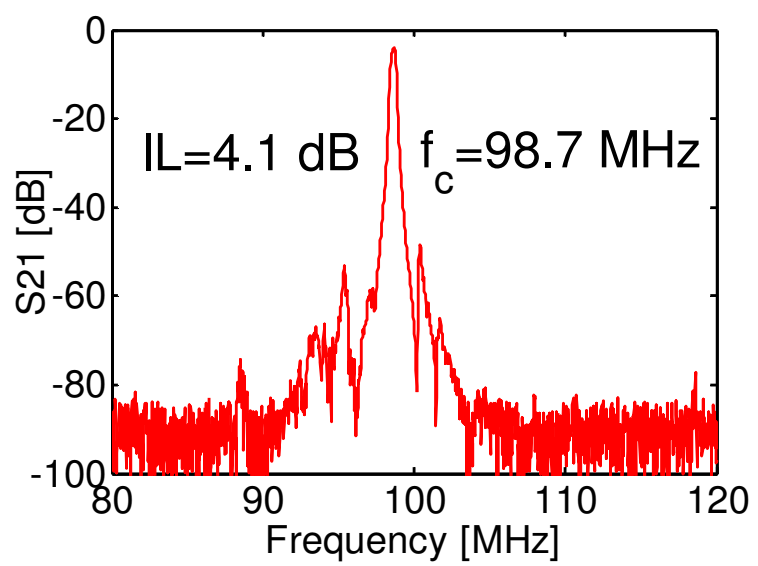

Figure 5. Performance of a $98.7 \mathrm{MHz}$ filter that was co-fabricated with the switches $($ Termination $=500 \Omega$ )

The three-finger dual-beam actuation switch showed low voltage actuation (15-30V). The RF performance of the switch was measured with the same network analyzer. Fig. 6 shows the performance of a $200 \times 200 \mu \mathrm{m}$ switch from 10 to 500 MHz. The separation of the RF and DC lines has reduced the parasitic loss in the switch and improved its performance. In the frequency range of interest for the switching of the fabricated filters, the switch shows isolation $>-50 \mathrm{~dB}$ up to $500 \mathrm{MHz}$. The insertion loss of the switch is higher than expected $(-0.7 \mathrm{~dB}$ at $500 \mathrm{MHz})$, but this is likely to be attributed to the mismatch losses in the switch layout and the high resistance of the contact materials (Platinum and NickelChrome alloy). A thin layer of nichrome that is used as the adhesion layer for the gold electroplating seed layer actually forms the contact with Platinum.

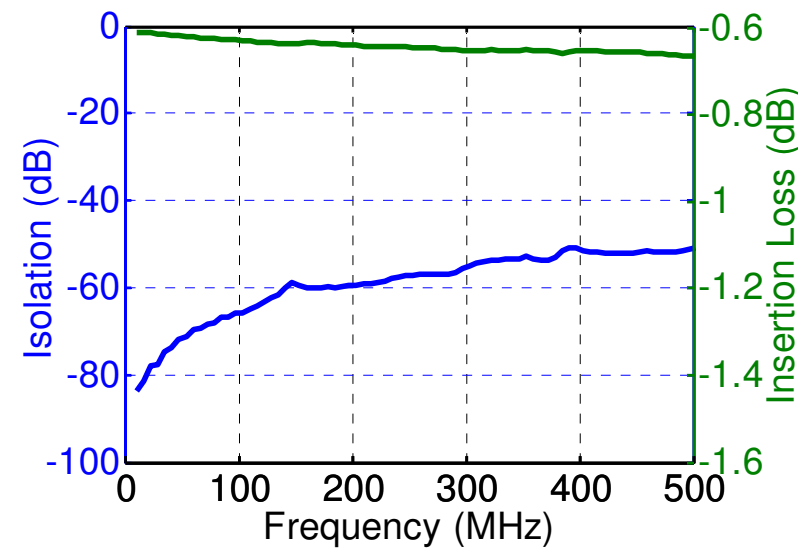

Figure 6. Measurement of isolation and insertion loss (IL) for a $200 \times 200 \mu \mathrm{m}$ three-finger dual-beam actuation AlN switch.

An interesting experimental aspect of the three-fingered dual-beam actuation switches that is worth reporting is that these devices have displayed very fast switching. They have consistently shown switching times $<1 \mu \mathrm{s}$. Fig. 7 shows the normalized response of a $300 \times 200 \mu \mathrm{m}$ switch that was actuated using a square waveform of magnitude $\pm 27.5 \mathrm{~V}$ at $100 \mathrm{~Hz}$. This switch has shown closure in approximately $230 \mathrm{~ns}$. The fast switching speed gives these switches a unique 
advantage over other MEMS implementation and makes possible the demonstration of other RF systems that rely on very fast reconfiguration requirements.

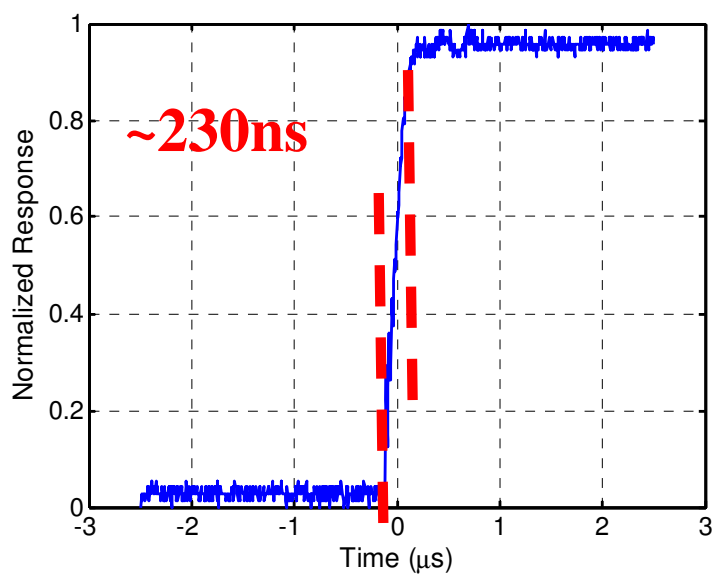

Figure 7. Response of the $300 \times 200 \mu \mathrm{m}$ switch to a square-wave form signal $( \pm 27.5 \mathrm{~V})$ at $100 \mathrm{~Hz}$

Fig. 8 shows the result of the co-integration of a single dual-beam switch with a third-order filter centered at 279.9 MHz. The plot shows that with just one switch in series with the filter, it is possible to effectively turn the filter on and off. When the switch is 'off' the response of the filter is lowered to $\sim-57 \mathrm{~dB}$, whereas when the switch is 'on' the insertion loss of the filter is $\sim-16.6 \mathrm{~dB}$ (as without the switch). The off-band rejection of the filter is $>50 \mathrm{~dB}$. This specific device that was co-fabricated with the switch does not attain the same filter performance that had been previously demonstrated. The reason for this degraded response (especially the IL) can be attributed to the limited yield of this specific fabrication run and not the integration per se. The demonstration can still be considered as a proof-of-concept of the monolithic integration of contour-mode filters with AlN switches and shows how the switch can effectively turn off the filter. New fabrication runs and testing are underway to demonstrate better performance of switchable filters.

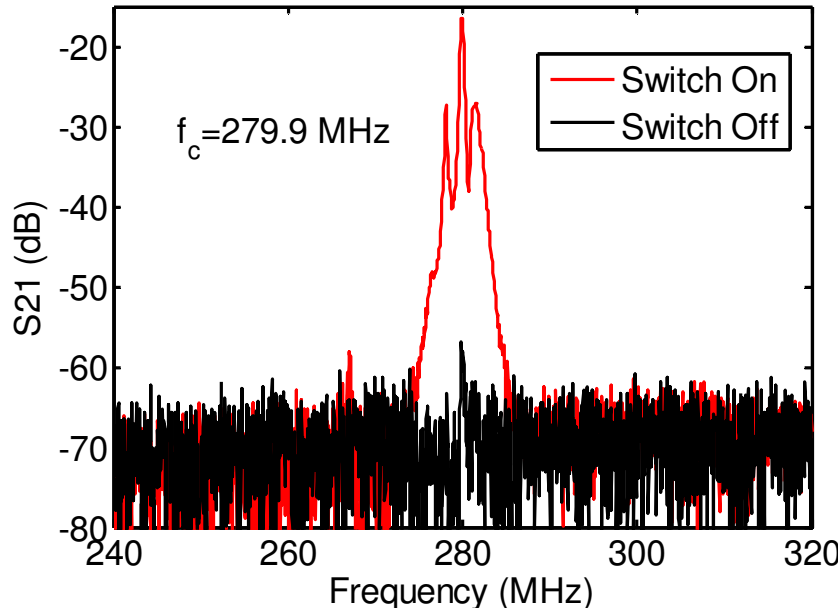

Figure 8. Response of a $279.9 \mathrm{MHz}$ filter with the switch in the on and off states. $($ Termination $=2 \mathrm{k} \Omega$ ).

\section{CONCLUSIONS}

This paper reports on the first demonstration of the integration of AlN based three-finger dual-beam actuation switches with contour-mode filters. This demonstration of allmechanical switchable AlN filters is the first step towards the realization of a highly reconfigurable RF architecture based on multi-frequency and multi-band standards. On-going research is focusing on improving the yield of the process and reducing the inductive loss in the switch.

\section{ACKNOWLEDGMENTS}

The authors will like to acknowledge the help of the staff of the Wolf Nanofabrication Facility at the University of Pennsylvania. The AlN films used were deposited by Tegal Corporation.

\section{REFERENCES}

[1] G. Piazza, P. J. Stephanou and A. P. Pisano, "Single-Chip MultipleFrequency AlN MEMS Filters Based on Contour-Mode Piezoelectric Resonators", Journal of MicroElectroMechanical Systems, 16/2/2007, pp. 319-328.

[2] C. Zuo, N. Sinha, C. R. Perez, R. Mahamameed, M. B. Pisani, and G. Piazza, "Hybrid Ultra-Compact 4th Order Band-Pass Filters Based On Piezoelectric AIN Contour-Mode MEMS Resonators," 2008 Solid-State Sensors, Actuators, and Microsystems Workshop, pp. 324-327, 2008.

[3] C. Zuo, N. Sinha, M. B. Pisani, C. R. Perez, R. Mahameed and G. Piazza, "Channel-Select RF MEMS Filters Based on Self-Coupled AlN Contour-Mode Piezoelectric Resonators," Ultrasonics Symposium, 2007. IEEE, vol., no., pp.1156-1159, 28-31 Oct. 2007.

[4] M. Rinaldi, C. Zuniga, C. Zuo and G. Piazza, "AlN contour-mode resonators for narrow-band filters above $3 \mathrm{GHz}$, Proceedings of Intl. Frequency Control Symposium 2009, In Press.

[5] G. Piazza, P. J. Stephanou and A. P. Pisano, "Piezoelectric Aluminum Nitride Vibrating Contour-Mode MEMS Resonators", Journal of MicroElectroMechanical Systems, 15/6/2006, pp. 1406-1418.

[6] G. Piazza, P. J. Stephanou and A. P. Pisano, "One and two port Piezoelectric Higher Order Contour-mode MEMS Resonators for Mechanical Signal Processing", Solid State Electronics, 51/ 1112/2007, pp. 1596-1608.

[7] M. Rinaldi, C. Zuniga and G. Piazza, "5-10 GHz AlN Contour-Mode Nanoelectromechanical Resonators", IEEE 22nd International Conference on MEMS 2009, pp. 916-919, 2009.

[8] G. M. Rebeiz, "RF MEMS Theory, Design and Technology", $1^{\text {st }}$ Edition, John Wiley \& Sons, Inc., 2003.

[9] H. C. Lee, J. H. Park, J. Y. Park, H. J. Nam and J. U. Bu "Design, fabrication and RF performances of two different types of piezoelectrically actuated Ohmic MEMS switches", J. Micromech. Microeng., 15, pp. 2098-2104.

[10] J. S. Pulskamp, D. C. Judy, R. G. Polcawich, R. Kaul, H. Chandrahalim and S. A. Bhave, "Monolithically Integrated Piezomems SP2T Switch and Contour-Mode Filters," IEEE 22nd International Conference on MEMS 2009, pp.900-903, 2009.

[11] N. Sinha, R. Mahamameed, C. Zuo, M. B. Pisani, C. R. Perez, and G. Piazza, 2008 Solid-State Sensors, Actuators, and Microsystems Workshop, pp. 22-25, 2008.

[12] R. Mahamameed, N. Sinha, M. B. Pisani, and G. Piazza, "Dual Beam Actuation of Piezoelectric AIN RF MEMS Switches Monolithically Integrated with AlN Contour-mode Resonators," J. Micromech. Microeng. 18, 105011, 2008.

[13] R. Ruby, P. Bradley, J. Larson III, Y. Oshmyansky and D. Figueredo, "Ultra-miniature high-Q filters and duplexers using FBAR technology ", IEEE Intl Dig. of Technical Papers. ISSCC. 2001, pp.120-121, 2001. 